\title{
8,12-Dibenzyl-4,16-dimethyl-1,3,8,12,17,18-hexaazatricyclo[12.2.1.1 $\left.{ }^{3,6}\right]$ octadeca- 4,6(18), 14(17), 15-tetraene
}

\author{
Farid Berhili ${ }^{\text {a }}$, Rachid Touzani ${ }^{a}$, Abdelkrim Ramdani ${ }^{a}$ and Sghir El Kadiri ${ }^{b_{*}}$ \\ a Laboratoire de Chimie Organique-Physique, Faculté des Sciences, Université Mohammed Ier Oujda, \\ Maroc \\ ${ }^{\mathrm{b}}$ Laboratoire de Chimie de l'Environnement et des Matériaux, Faculté des Sciences, Université \\ Mohammed Ier Oujda, Maroc \\ * Fax : +21256500603 E-mail: elkadiri@sciences.univ-oujda.ac.ma
}

Received: 16 June 2002 / Accepted: 30 September 2002 / Published: 11 March 2003

Keywords: macrocyclic compounds, macrocyclic cavity, pyrazole<smiles>Cc1cc(CCl)nn1Cn1nc(CCl)cc1C</smiles>

1

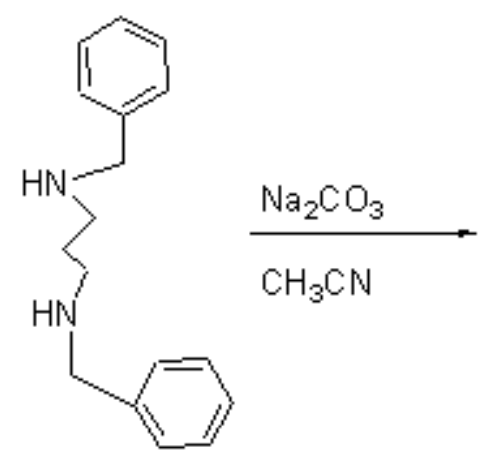

2<smiles>Cc1cc2nn1Cn1nc(cc1C)CN(Cc1ccccc1)CCCN(Cc1ccccc1)C2</smiles>

3

A suspension of sodium carbonate $(12 \mathrm{~g}, 120 \mathrm{mmol})$ in acetonitrile $(250 \mathrm{~mL})$ was refluxed under magnetic stirring, then a solution of bis-(3-chloromethyl-5-methylpyrazolyl)methane (1) $(2 \mathrm{~g}, 7 \mathrm{mmol})$ [1, 2] and diamine $2(1.68 \mathrm{~g}, 7 \mathrm{mmol})$ in acetonitrile $(50 \mathrm{~mL})$ was added dropwise. The solution was refluxed under stirring for two hours, filtered and the solvent was removed in vacuum, the residue was purified on alumina column with $\left(\mathrm{CH}_{2} \mathrm{Cl}_{2} / \mathrm{MeOH}: 95 / 5\right)$ as eluent to give the macrocycle 3 as an oily substance.

Yield: $(1.5 \mathrm{~g}, 55 \%)$.

$(\mathrm{FAB})^{+}[\mathrm{M}+\mathrm{H}]^{+}=457$.

${ }^{1} \mathrm{H}$ NMR (250 MHz, $\mathrm{CDCl}_{3}$ ) d ppm: 7.36 (m, 10H, Ph) ; 5.91 (s, 2H, CH2-NPz) ; 5.86 (s, 2H, HPz) ; 3.60 (s, 4H, N- $\left.\underline{\mathrm{CH}}_{2}-\mathrm{Ph}\right) ; 3.41$ (s, 4H, Pz-CH $\left.2-\mathrm{N}\right) ; 2.30$ (s, 6H, CH3 $\left.-\mathrm{Pz}\right) ; 2.20$ (t, 4H, N- $\left.\underline{\mathrm{CH}}_{2}-\mathrm{CH}_{2}\right) ; 1.30$ (m, $\left.2 \mathrm{H}, \mathrm{CH}_{2}-\mathrm{CH}_{2}-\mathrm{CH}_{2}\right)$.

\section{References}

1. El Kadiri, S.; Ph. D. Thesis, U. S. T. L. Montpellier, 1988.

2. Malek, F.; Persin, M.; Ramdani, A.; Sarrasin, J.; Zidane, I. New. J. Chem. 2002, 2, 26.

(C) 2003 MDPI. All rights reserved. 